\title{
Multiple Embolic Infarction Due to a Primary Aortic Intimal Sarcoma
}

\author{
Heejung Mo, ${ }^{\mathrm{a}, \mathrm{b}}$ Hyung-Min Kwon, ${ }^{\mathrm{a}}$ Jae-Sung Choi, ${ }^{\mathrm{c}}$ Seon Jae Ahn, ${ }^{\mathrm{a}, \mathrm{b}}$ Yong-Seok Lee ${ }^{\mathrm{a}}$ \\ aDepartment of Neurology, SMG-SNU Boramae Medical Center, Seoul National University College of Medicine, Seoul, Korea \\ 'Department of Neurology, Seoul National University Hospital, Seoul, Korea \\ 'Department of Thoracic and Cardiovascular Surgery, SMG-SNU Boramae Medical Center, Seoul National University College of Medicine, \\ Seoul, Korea
}

\section{Dear Sir:}

Primary aortic sarcoma is a very rare but aggressive tumor with frequent metastasis to bone and visceral organs.' There have been less than 40 reported cases of primary aortic intimal sarcoma. The most common locations of the tumor are the abdominal aorta (40\%), thoracic aorta (20\%), and thoracoabdominal aorta (10\%). ${ }^{1-3}$ Most clinical presentations are embolic phenomena or vessel occlusion or obstruction, i.e. claudication or abdominal pain rather than symptoms directly related to the primary tumor. Cerebral infarction is an extremely rare presentation of sarcoma. ${ }^{1.4,5}$

Here, we report a case of primary intimal sarcoma of the aortic arch and ischemic stroke.

A 41-year-old woman visited the emergency room for vertigo one hour after onset. She was an ex-smoker with a 30 pack-year history and a social drinker. She had no known hypertension, diabetes, cardiac disease or previous stroke. On initial neurological evaluation, she had no definite abnormalities including ocular movements and gaze. She was afebrile and her blood pressure was $119 / 72 \mathrm{mmHg}$; her hemoglobin level was $13.3 \mathrm{~g} / \mathrm{dL}$.

Brain MRI revealed multiple tiny infarctions in the right cerebellar hemisphere and left parietal cortex. MR angiography of the intracranial and neck vessels showed no steno-occlusive lesions (Figure 1A). Initial serum D-dimer was $1.30 \mathrm{mg} / \mathrm{L}$ (reference: 0-0.4 mg/L). Carotid duplex sonography did not show any abnormalities. Contrast-enhanced computed tomography angiography showed a mass-like lesion at the ascending aorta sus- pected of a mural thrombus; there was no wall thickening, wall enhancement, stenosis or aneurysmal dilatation in the ascending aortic lesion (Figure 1B).

After admission, she experienced severe abdominal pain. From CT angiography, we found splenic infarction and a left subclavian thrombus. Anticoagulant therapy was started immediately because a mural aortic thrombus was suspected to be the source of the distal embolization. However, she had recurrent abdominal pain, and follow-up abdominal CT angiography revealed another embolic infarction at the right kidney. Transthoracic echocardiography showed a large hypermobile mass attached to the ascending aorta (Figure 1C). MRI of the heart showed an elongated lobulated mass in the ascending thoracic aorta, attached to the left lateral wall, suggesting angiosarcoma (Figure 1D).

Because of the recurrent embolic events, she was transferred to the department of thoracic surgery for palliative tumor removal. The tumor was $1 \times 2 \mathrm{~cm}$ and there was no evidence of a thrombus in the specimen. Histopathological examination was consistent with myxosarcoma that stained positive for vimentin (Figure 1E). The patient started palliative chemotherapy with doxorubicin followed by pazopanib. She was discharged without acute complications. Six months later, she admitted to the department of oncology due to the bone metastasis. After palliative care for 1 month, she transferred to the hospice palliative care center.

Our patient was a heavy ex-smoker and the initial CT angiography finding was suggestive of a mural thrombus of the aorta. So, the stroke could be explained by embolism due to aortic 

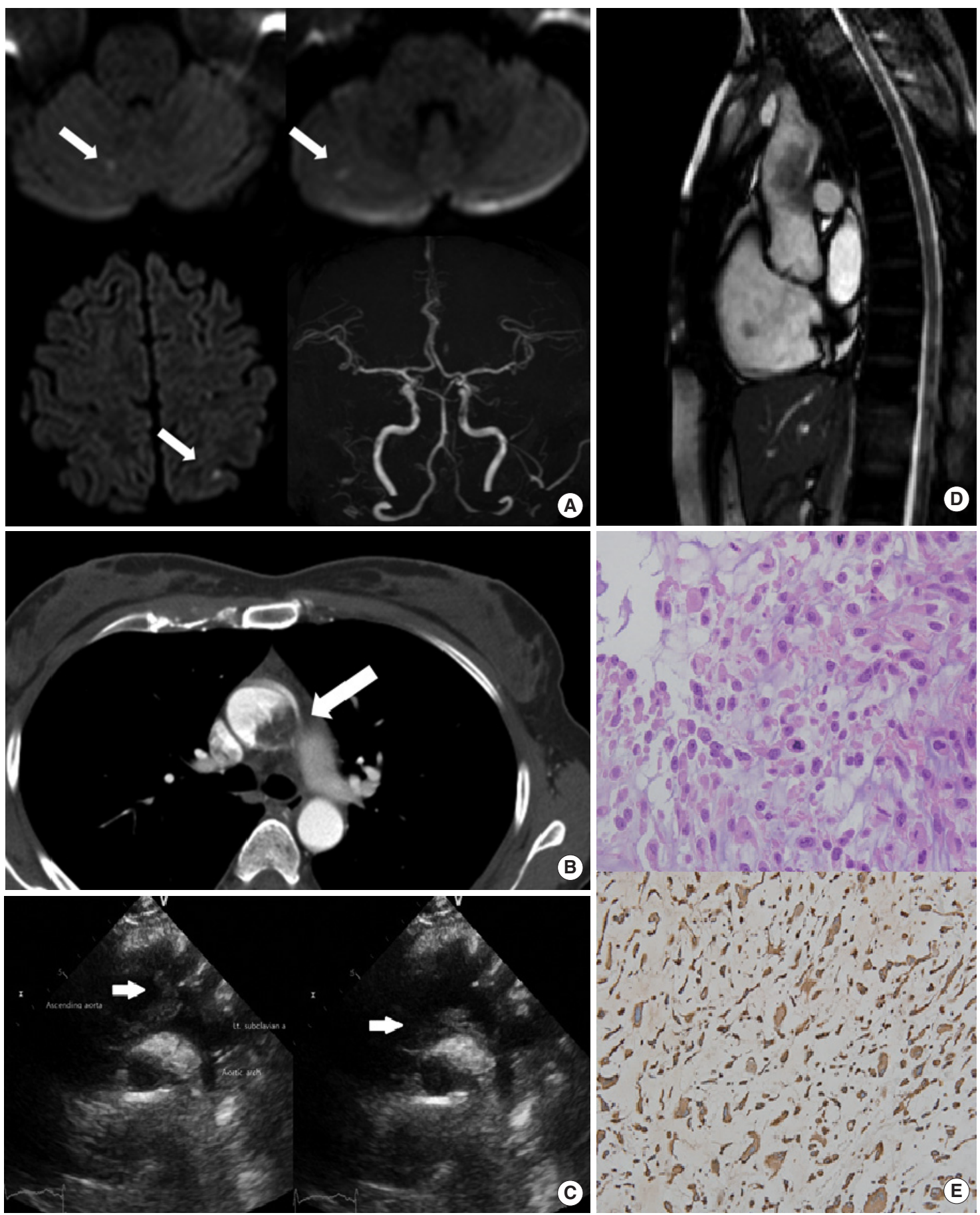

Figure 1. (A) Brain MRI with diffusion-weighted imaging showed multiple embolic infarctions of right cerebellum and left parietal cortex (arrows), and MR angiography of the brain revealed no definite abnormality. (B) Computed tomography imaging with contrast showed a space-occupying substance, annotated with arrow (interpreted by the radiologist as ascending aortic thrombus). (C) Transthoracic echocardiography showed a large hypermobile mass (white arrows) attached to the ascending aorta. (D) MRI of the heart revealed a mass-like lesion in the ascending aortic arch. (E) Atypical spindle cells with irregular and hyperchromatic nuclei were seen with hematoxylin and eosin staining $(\times 200)$. Immunohistochemical staining for vimentin was positive $(\times 200)$.

thrombus. However, ex-smoking alone cannot be the cause of a large thrombus in the ascending aorta. The patient experienced multiple embolic infarctions throughout the brain and visceral organs during admission and the ascending aorta was not the common location of thrombus formation; there was no evidence of hypercoagulability or other thrombi in the neighboring transverse or descending aorta.

These clinical findings strongly suggested an embolic mecha- 
nism outside of conventional etiology. From transthoracic echocardiography and MRI of the heart, we concluded that recurrent embolic events were due to tumor emboli of the intimal aortic sarcoma.

The diagnosis of aortic sarcoma is challenging. Through previous case reports, aortic sarcoma often presents similarly to an atherosclerotic plaque in computed tomography imaging. MRI of the heart can differentiate between thrombus and tumor by heterogeneity on T1-weighted images and hyperintensity on T2weighted images, delayed enhancement with gadolinium or 'cauliflower' appearance. Therefore, some studies suggest that MR with contrast imaging may be the method of choice. ${ }^{6,7}$

The prognosis of this tumor is dismal because of the advanced stage at which the disease is diagnosed in most cases. Mean survival period of patients with an established diagnosis is around 16 months (range: 0-168 months), and the 3- and 5-year survival rates are about $11.2 \%$ and $8 \%$, respectively. ${ }^{3.6}$ Therefore, clinicians must be aware of intimal aortic sarcoma in patients with recurrent emboli and unusual location of thrombi in the aortic arch.

\section{References}

1. Sebenik M, Ricci A Jr, DiPasquale B, Mody K, Pytel P, Jee KJ, et al. Undifferentiated intimal sarcoma of large systemic blood vessels: report of 14 cases with immunohistochemical profile and review of the literature. Am J Surg Pathol 2005;29:11841193.

2. Staats P, Tavora F, Burke AP. Intimal sarcomas of the aorta and iliofemoral arteries: a clinicopathological study of 26 cases. Pathology 2014;46:596-603.

3. Scharl $M$, Bode $B$, Rushing $E$, Knuth $A$, Rordorf T. Uncommon case of brain metastasis in a patient with a history of heavy smoking. Curr Oncol 2014;21:e728-e731.

4. Seelig MH, Klingler PJ, Oldenburg WA, Blackshear JL. Angiosarcoma of the aorta: report of a case and review of the literature. J Vasc Surg 1998;28:732-737.

5. Nan YY, Liu YC, Lu MS, Hsueh S, Chang HK, Huang YK. Angiosarcoma in the aortic arch presented as repeat strokes. J Thorac Cardiovasc Surg 2010;139:e40-e42.

6. Brylka D, Demos TC, Pierce K. Primary angiosarcoma of the abdominal aorta: a case report and literature review (aortic angiosarcoma). Abdom Imaging 2009;34:239-242.

7. Riles E, Gupta S, Wang DD, Tobin K. Primary cardiac angiosarcoma: a diagnostic challenge in a young man with recurrent pericardial effusions. Exp Clin Cardiol 2012;17:39-42.

Correspondence: Hyung-Min Kwon Department of Neurology, Seoul Metropolitan Government-Seoul National University, Boramae Medical Center, Seoul National University College of Medicine, 20 Boramae-ro 5-gil, Dongjak-gu, Seoul 07061, Korea

Tel: $+82-2-870-2475$

Fax: +82-2-831-2826

E-mail:hmkwon@snu.ac.kr

Received: April 18, 2016

Revised: May 9, 2016

Accepted: June 23, 2016

The authors have no financial conflicts of interest. 